\title{
Atypical Hemolytic Uremic Syndrome During Pregnancy: Short Review and Case Report with a Favorable Outcome
}

\author{
N.A.Zharkin ${ }^{*}$, M.E.Statsenko ${ }^{1}$, M.M.Stazharova², N.A.Burova1 ${ }^{1}$, S.A.Prokhvatilov², N.E.Kushniruk ${ }^{2}$ \\ ${ }^{1}$ Department of Obstetrics and Gynecology, Department of Internal Diseases of Pediatric Faculty of Volgograd State \\ Medical University, Volgograd, Russia. \\ ${ }^{2}$ Department of Nephrology, Department of Gynecology, VRCH No. 1, Volgograd Regional Clinical \\ Hospital No.1), Volgograd, Russia.
}

*Corresponding Author: N.A.Zharkin, Department of Obstetrics and Gynecology, Department of Internal Diseases of Pediatric Faculty of Volgograd State Medical University, Volgograd, Russia; E-mail: zharkin55@mail.ru

Received: 23 March 2018; Accepted: 09 April 2018; Published: 10 April 2018

\begin{abstract}
The atypical hemolytic uremic syndrome (aHUS) is the most complex disease in the diagnosis, treatment and prognosis among the thrombotic microangiopathy. The article describes the clinical case of a 32-year-old woman with an aHUS in 32 week of gestation. It is given the diagnosis and treatment of this case till clinical convalescence. Observation within 1 year after the episode of the aHUS is showing up a chronic kidney failure, which will provide renal replacement therapy in future.
\end{abstract}

Keywords: Atypical Hemolytic Uremic Syndrome; Acute Renal Failure; Complement Pathway; Chronic; Plasma Exchange; Postpartum Period; Pregnancy; Chemotactic Factor Inactivator; Complement; Eculizumab; Renal Dialysis; Thrombotic Microangiopathy.

\section{Case}

Thrombotic microangiopathy (TMA) is a group of diseases associated with hemolysis, hemorrhagic syndrome and acute renal failure. During pregnancy, these diseases cause high maternal and perinatal morbidity and mortality due to the difficulty of diagnosing and different approaches to treatment, depending on the TMA type. 
Recently, cases of TMA in pregnant women due to sometimes dramatic events have been reported more frequently, that is why each case with a favorable outcome for the mother and her child can be an important matter for discussion and enrichment of the general knowledge bank with data on this rare but life-threatening pathology.

There are several variants of TMA that complicate the course of pregnancy: pregnancy-associated hypertension, preeclampsia (PE), DIC and HELLP syndrome, thrombotic thrombocytopenic purpura (TTP), catastrophic antiphospholipid syndrome (CAS), sepsis and, as the main manifestation of TMA, - atypical hemolytic uremic syndrome (aHUS) [1,2]. Life-threatening complications of TMA include acute renal failure (ARF), brain injury (coma), gastrointestinal tract injury (hemorrhage), and heart failure (acute heart failure). Predisposing factors of TMA are preeclampsia, premature birth, caesarean section, infection, psycho-traumatic situations $[3,4]$.

Diagnosing and treatment of various variants of TMA in pregnant women involves considerable difficulties. That is why, needless to say that colleagues wish to share their experience on each medical case with both favorable $[4,5]$ and unfavorable outcomes [6].

Among TMA, atypical hemolytic uremic syndrome (aHUS) is the most complicated disease in terms of diagnosing, treatment and prognosis [7]. It is caused by genetic defects in regulatory proteins of the complement system. In this regard, as opposed to preeclampsia and HELLP-syndrome, childbirth does not lead to the regression of symptoms; on the contrary, the microangiopathic process worsens with rapid development of multiple organ failure.

Currently, there is no doubt that preeclampsia and HELLP-syndrome are connected with genetically determined dysregulation of the alternative complement pathway (ACP), which may become excessive, uncontrolled during pregnancy, which, probably, may contribute to the early-onset severe preeclampsia with subsequent transformation to aHUS $[8,9]$.

The confounding factor is that these genetic disorders in the complement system manifest for the first time precisely during pregnancy or childbirth, which causes the need to perform diagnosis and choose treatment policy within a limited time period. Our medical case confirms this as well.

A 32-year-old patient with second pregnancy, first delivery. She had no diseases before pregnancy. In 2015, the first pregnancy ended with an abortion at her will. The second pregnancy was uneventful until 33 weeks. Initial laboratory blood and urine values were normal: hemoglobin $132 \mathrm{~g} / \mathrm{L}$, platelets $214 \times 10^{9}$. Common urine test has not detected protein; urinary sediment was empty.

Ultrasound screening at 33 weeks revealed fetoplacental insufficiency and fetal intrauterine growth retardation (IUGR), and therefore the patient was hospitalized for examination and treatment. Clinical and laboratory examination revealed no abnormalities. In connection with IUGR and the perspective of preterm childbirth, prevention of RDS (respiratory distress syndrome) of the fetus with corticosteroids was carried out. 
On $08 / 28 / 2016$ at 11:10, a partial abruption of a normally situated placenta with external hemorrhage of approximately $200 \mathrm{~mL}$ occurred. In this regard, the emergency caesarean section was performed. A girl was born with mass of $1440 \mathrm{~g}$, height of $40 \mathrm{~cm}, 4$ and 6 scores by the Apgar scale. The total blood loss volume was $1250 \mathrm{~mL}$ : before the surgery - $200 \mathrm{~mL}$, the volume of the retroplacental hematoma $-400 \mathrm{~mL}$, during the surgery $-650 \mathrm{~mL}$. At the end of the surgery, hemoglobin amounted to $78 \mathrm{~g} / \mathrm{L}$, platelets $-170 \times 10^{9}$.

Within the first 24 hours, the post-surgery period was complicated by the development of anuria, emergence and rapid augmentation of azotemia (serum creatinine amounted to 188 - $203 \mu \mathrm{mol} / \mathrm{L}$ ), which allowed to establish acute renal failure. The patient was treated with infusions of crystalloids, fresh frozen plasma, and furosemide for diuresis stimulation.

On the following day, 08/29/2016, negative dynamics was observed: pale skin and swelled face, lower limbs and the anterior abdominal wall. Respiration rate -24 breaths per minute. Vesicular breathing in the lungs, no crackles. Heart sounds were rhythmic, muffled. Heart rate -76 beats per minute, blood pressure $-130 / 90 \mathrm{~mm} \mathrm{Hg}$. The progression of anemia was observed $(\mathrm{Hg}=68 \mathrm{~g} / \mathrm{L})$, as well as a decrease in platelets up to $150 \times 10^{9}$ and a rise in blood creatinine up to $342 \mu \mathrm{mol} / \mathrm{L}$. Anuria persisted.

Laboratory tests showed rapidly increasing anemia $(\mathrm{HB}=63-58 \mathrm{~g} / \mathrm{L})$ and thrombocytopenia up to $50 \times 10^{9}$, single schizocytes. Blood chemistry: creatinine $404-611 \mu \mathrm{mol} / \mathrm{L}$, AST - $205 \mathrm{U} / \mathrm{L}$, ALT - $345 \mathrm{U} / \mathrm{L}$, Potassium - 5.17 mmol/L, Sodium - 132 mmol/L, LDH - 2996 U/L, Coombs’ test negative.

Ultrasound of the abdominal cavity and kidneys: the liver and spleen are not changed, the urinary bladder is empty, the size of the kidneys is not changed, the parenchyma with increased echogenicity, the corticomedullary differentiation is pronounced; during $\underline{\mathrm{CDI}}$ (Color Doppler Imaging), the vascularization is visible up to the level of the arcuate vessels. Chest X-ray: lung pattern without focal and infiltrative shadows. There was a small amount of fluid in the lower parts of the pleural cavities. Transfusion of washed red blood cells, antibacterial and detoxification therapy were added to the treatment regimen.

Considering the rapidly increasing anemia in the absence of hemorrhage with signs of microangiopathic hemolysis (high LDH values), thrombocytopenia, acute renal failure (anuria, rapid increase of creatinine), TMA was suspected, namely, atypical hemolytic uremic syndrome.

To verify the diagnosis, a test for ADAMTS-13 was performed, the level of which amounted to $40 \%$ (reference values $93-113 \%$ ), which allowed to establish the final diagnosis of aHUS. Specialists from the Federal Center gave consultation via videoconferencing.

On 08/31/2016, the treatment with fresh frozen plasma was started in plasma-exchange regimens in the volume of $30 \mathrm{~mL} / \mathrm{kg}$ of body weight per day (in total - 4 procedures) under the cover of unfractionated heparin (UFH) in a dose 
of $70-80 \mathrm{mg} / \mathrm{kg}$ of weight. Renal replacement therapy was started in the form of prolonged veno-venous hemodiafiltration sessions (PVVHDF). In total, 5 sessions were performed during the hospitalization.

Arterial hypertension appeared during the following days (blood pressure 150/90 - 170/100 mm $\mathrm{Hg}$ ), for which reason the therapy was supplemented with antihypertensive drugs. Echocardiography showed concentric remodeling of the left ventricle myocardium, normal systolic and diastolic functions of the left ventricle, the ejection fraction was $62 \%$.

Despite the therapy, the signs of ongoing microangiopathic hemolysis were observed in the patient: persisting anemia $(\mathrm{HB}=56$ - $65 \mathrm{~g} / \mathrm{L})$, thrombocytopenia (plt=77 - 80x1099), high LDH values (1269 - 714 U/L), lack of resolution of acute renal failure, persisting azotemia (blood creatinine $=516-836 \mu$ mol/L). Skin hemorrhagic syndrome appeared with a massive hemorrhagic rash on the body, upper and lower limbs. Swelling of the face and the lower limbs increased. Weakening of breathing was observed in the lower sections of the lungs. There was persistent hypertension in the range from $150 / 90$ to $200 / 130 \mathrm{~mm} \mathrm{Hg}$.

On $09 / 02 / 16$, on the $5^{\text {th }}$ day of the patient's stay in the hospital, after the second remote consultation with specialists from Moscow, it was decided to cancel the plasma-exchange sessions and to start targeted therapy with Eculizumab (Soliris), at a dose of $900 \mathrm{mg}$ I.V. by drop infusion, once a week, a total of 4 infusions.

After 4 weeks, the patient's condition improved: peripheral and cavitary swelling regressed, hemorrhagic syndrome was neutralized, acute renal failure was resolved, diuresis recovered after the $2^{\text {nd }}$ infusion of Eculizumab on the $17^{\text {th }}$ day of the anuretic period. Blood pressure decreased to $140 / 90 \mathrm{~mm} \mathrm{Hg}$.

Laboratory tests showed an improvement of anemia $(\mathrm{HB}=109 \mathrm{~g} / \mathrm{L})$; the signs of microangiopathic hemolysis were neutralized (platelets increased up to $180 \times 10^{9}$ ), LDH decreased to $341 \mathrm{U} / \mathrm{L}$, the values of nitrogen metabolism improved significantly, serum creatinine decreased to $219 \mu \mathrm{mol} / \mathrm{L}$.

With repeated dynamic nephrosonographies after administration of Eculizumab, we observed a weakened blood flow above the arcuate arches in the both kidneys. On echocardiography, the ejection fraction increased up to $70 \%$. Therapy with Eculizumab was cancelled. On 09/29/2016, the patient was discharged with recommendations to continue nephrotoprotective therapy.

During a year after discharge, the patient was followed-up with monthly laboratory testing. The results are presented in the chart. Nephrosonography performed in December 2017 showed signs of decreasing size of the kidneys and signs of developing nephrosclerosis. 


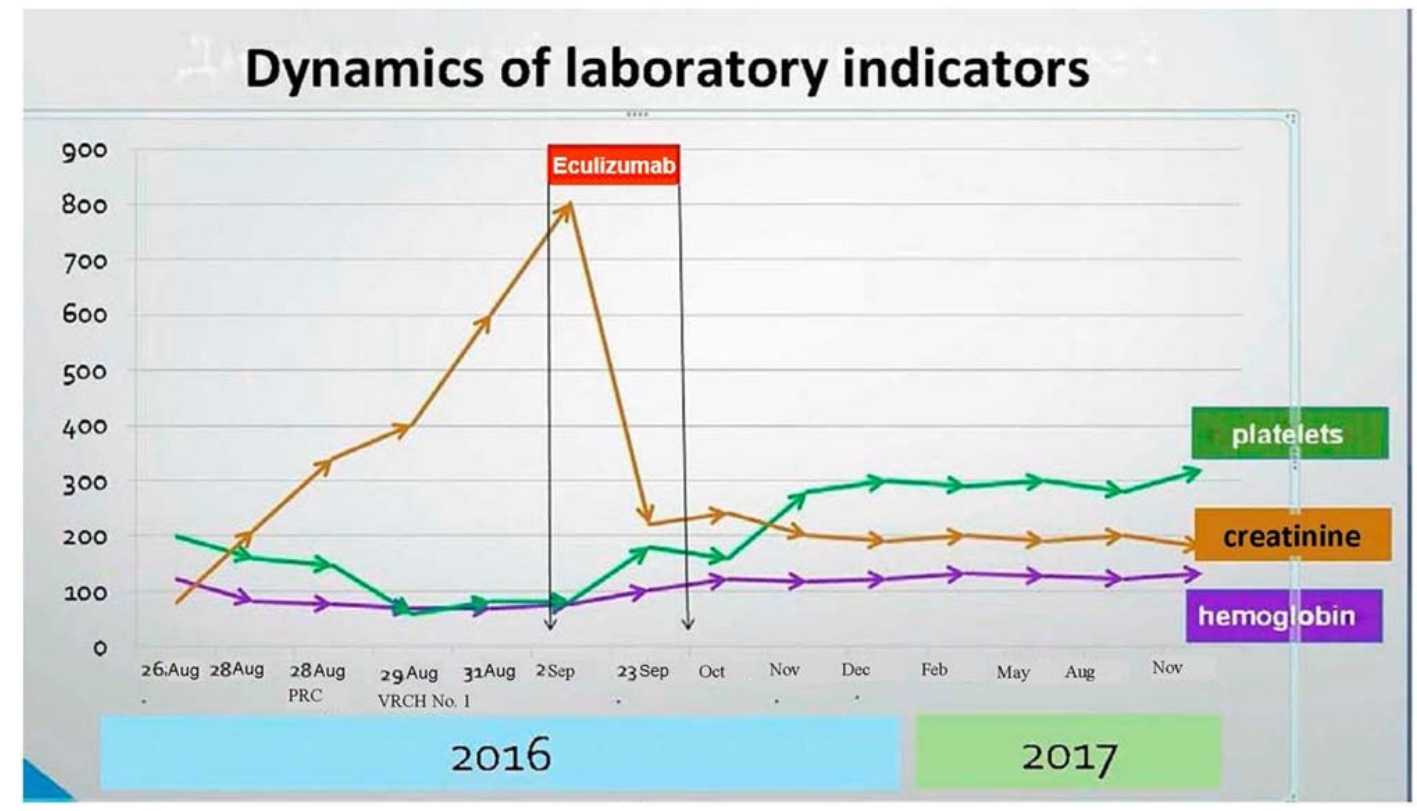

\section{Discussion}

The present observation demonstrates the peculiarities of clinical manifestation and the course of "obstetric" aHUS with onset in the postpartum period. The sum of various obstetric complications, namely, the combination of a premature placental abruption with hemorrhage, caesarean section, postpartum inflammation caused by a large wound surface in the uterus, led to systemic activation of the alternative complement pathway, which induced the development of aHUS in the patient with a genetic predisposition.

A typical clinical and laboratory pattern of TMA involves primary renal damage in the onset of the disease, development of microangiopathic hemolytic anemia and thrombocytopenia, decreased level of ADAMTS-13, which indicates active formation of microthrombi [10]. Detection of these symptoms in progress allowed us to establish aHUS in this patient.

Despite a moderate decrease in the severity of hemolysis, therapeutic plasma exchange started on the $3^{\text {rd }}$ day of the postpartum period had not led to the expected results. This fact required administration of targeted therapy with complement-blocking drug Eculizumab. It is a glycosylated, humanized monoclonal antibody, a kappaimmunoglobulin with high affinity for the complement component C5. Through binding to the latter, Eculizumab prevents further activation of the complement by stopping its destroying activity. By the end of induction therapy consisting of 4 infusions with a week's interval, it was possible to stop the hematological signs of TMA.

Performed sessions of hemodiafiltration contributed to the resolution of acute renal failure. Although the renal function had not fully recovered by the time of patient's discharge, any further renal replacement therapy sessions were not required. 
The follow-up showed that despite complete hematological remission of aHUS and nephroprotective therapy, the patient still demonstrated signs of renal failure. A chronic renal disease has formed. Nephrosclerosis (renal scarring) is developing, which determines an unfavorable outcome in terms of renal failure progression up to the terminal stage and further transition of the patient to renal replacement therapy in the long term.

\section{References}

1. Elayoubi J, Donthireddy K, Nemakayala DR. Microangiopathies in pregnancy. BMJ Case Rep 2018.

2. Silver RM. Catastrophic antiphospholipid syndrome and pregnancy. Semin Perinatol 42 (2018): 23-32.

3. Fox L, Cohney SJ, Kausman JY, Shortt J, Hughes PD, et al. Consensus Opinion on Diagnosis and Management of Thrombotic Microangiopathy in Australia and New Zealand. Nephrology (Carlton) 2018.

4. Valter Romão de Souza, Jr Ana Beatriz Cavalcante de Oliveira, Ana Maria Vanderlei, Amanda Queiroz da Mota Silveira Aroucha, Bruna Pontes Duarte, et al. Inherited thrombotic thrombocytopenic purpura mimicking immune thrombocytopenic purpura during pregnancy: a case report. J Med Case Report 2018.

5. Cravero R, Ardissino G, Colageo U, Staffa P, Bruschetta E, et al. Efficacy of eculizumab in a case of pregnancy-associated aHUS. Ital Nefrol 33 (2016): 14.

6. Pronina I S. Trudniy diagnoz: aHUS, assotsiirovaniy c rodami. Bulletin of Medical Internet Conferences 2016.

7. Zephirova TP, Maltseva LI, Zamaleeva RS, Zhelezova ME. Syndrome of thrombotic microangiopathy in life-threatening complications of pregnancy and labor in women. Prakticheskaya meditsina 7 (2017): 2630 .

8. Skully M. Thrombotic Thrombocytopenic Purpura and Atypical Hemolytic Uremic Syndrome Microangiopathy in Pregnancy. Semin_Thromb_Hemost 42 (2016):774-779.

9. Bruel A, Kavanagh D, Noris M, Delmas Y, Wong EKS, et al. Hemolytic Uremic Syndrome in Pregnancy and Postpartum. Clin J Am Soc Nephrol 12 (2017):1237-1247.

10. Cines DB, Levine LD. Hematology Thrombocytopenia in pregnancy. Hematology Am Soc Hematol Educ Program 2017 (2017): 144-151.

Citation: N.A.Zharkin, M.E.Statsenko, M.M.Stazharova, N.A.Burova, S.A.Prokhvatilov, N.E.Kushniruk. Atypical Hemolytic Uremic Syndrome During Pregnancy: Short Review and Case Report with a Favorable Outcome. Obstetrics and Gynecology Research 1 (2018): 021-027.

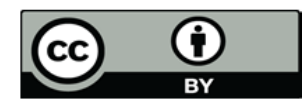

This article is an open access article distributed under the terms and conditions of the Creative Commons Attribution (CC-BY) license 4.0 